\title{
The behavioural and neurophysiological modulation of microsaccades in monkeys
}

\author{
Donald C. Brien \\ Queen's University \\ Brian D. Corneil
University of Western Ontario \\ Andrew H. Bell \\ Queen's University \\ Jillian H. Fecteau \\ Queen's University \\ Douglas P. Munoz \\ Queen's University
}

\begin{abstract}
Systematic modulations of microsaccades have been observed in humans during covert orienting. We show here that monkeys are a suitable model for studying the neurophysiology governing these modulations of microsaccades. Using various cue-target saccade tasks, we observed the effects of visual and auditory cues on microsaccades in monkeys. As in human studies, following visual cues there was an early bias in cue-congruent microsaccades followed by a later bias in cue-incongruent microsaccades. Following auditory cues there was a cue-incongruent bias in left cues only. In a separate experiment, we observed that brainstem omnipause neurons, which gate all saccades, also paused during microsaccade generation. Thus, we provide evidence that at least part of the same neurocircuitry governs both large saccades and microsaccades.
\end{abstract}

Keywords: covert orienting, cue-target task, omnipause neurons, fixational eye movements, crossmodal attention, oculomotor control

\section{Introduction}

Small, involuntary and unperceivable eye movements occur during visual fixation and are classified into three types: tremor, drift and microsaccades (Barlow, 1952; Ratliff and Riggs, 1950; Steinman, Haddad, Skavenski, and Wyman, 1973; Martinez-Conde et al., 2004). Although there is some debate as to their function (see Rolfs, 2009 and Martinez-Conde et al., 2009 for reviews), it appears that these fixational eye movements serve as a means of introducing stochastic displacement to the retinal image in order to aid in counteracting retinal adaptation (Ditchburn et al., 1959; Martinez-Conde et al., 2006b; Troncoso et al., 2008). If all eye movements were suppressed while viewing a visual scene, the image on the retina would eventually fade away (Riggs, et al.,
1953) in about 80ms (Coppola and Purves, 1996) and these small eye movements prevent this from happening.

Among these small eye movements, microsaccades produce the largest displacements and are therefore the easiest to study. These eye movements have the same velocity amplitude relationship as larger saccades (Zuber et al., 1965). The study of microsaccade metrics in humans has revealed that biases in the inherent randomness of microsaccades can be produced under certain conditions and these biases may reveal underlying neurophysiological processes (Hafed and Clark, 2002; Engbert and Kliegl, 2003; Galfano et al., 2004; Laubrock et al., 2005; Rolfs et al., 2004; Rolfs et al., 2005; Martinez-Conde and Macknik, 2007).

The pattern of microsaccades is biased in covert orienting tasks. Where our eyes are oriented (overt attention) and where our attention is actually focused (covert 
attention) can be separated with cueing tasks (Posner, 1980). A visual cue can be presented during fixation in a predictive (in the same location) or non-predictive (in a diametrically opposite location) location of an upcoming target. By reducing the time between the cue and the target (the CTOA - cue-target onset asynchrony), shorter reaction times to cue congruent targets were observed. This has been referred to as reflexive capture of attention (Jonides, 1981; Posner and Cohen, 1984; Remington et al., 1992). This early capture of attention is followed by a later epoch that results in longer reaction times to cue congruent targets and this has been referred to as inhibition of return (IOR) (Posner and Cohen, 1984; Posner et al., 1985; Maylor and Hockey 1985).

In human studies, it has been observed that microsaccade properties are modulated during these cueing tasks, but the modulation varies depending on the type of cue and experimental conditions used. First, microsaccade rate is affected by the sudden onset of both auditory and visual cues (Engbert and Kliegl, 2003; Rolfs et al., 2005). During steady fixation of a fixed visual display, microsaccades have a baseline rate of around one per second, but following the appearance of a cue in the periphery there is an early decrease in frequency of microsaccades around $150 \mathrm{~ms}$ after cue onset, followed by a later increase in frequency of microsaccades that peaks at twice that of baseline around $350 \mathrm{~ms}$ after cue onset. The early decrease in frequency was expected as this is also seen for larger saccades (Reingold and Stampe, 2002), but the later increase is novel to microsaccades (Engbert and Kliegl, 2003). Second, many studies have shown that microsaccade direction is affected by cue location (Hafed and Clark 2002; Engbert and Kliegl, 2003; Galfano et al., 2004; Laubrock et al., 2004; Rolfs et al., 2004; Rolfs et al., 2005). Following a peripheral cue, microsaccade directions are first biased towards the cue during the period of capture of attention and then biased away from the cue during the period of inhibition of return (Hafed and Clark, 2002; Galfano et al., 2004; Laubrock et al., 2004; Rolfs et al., 2004; Rolfs et al., 2005.) The magnitude of the influence of microsaccades on behavior is still debated (Horowitz et al., 2007a, Horowitz et al., 2007b and Laubrock et al., 2007) and appears to be much smaller and more complicated (Kliegl et al., 2009), than the influence of the cue. However, because the stochastic nature of microsaccades can be biased by spatial attention, the connection of attention to saccade programming is reinforced and they present an opportunity to study the underlying oculomotor processing that occurs during covert orienting.

As we attempt to understand the neurophysiology that underlies microsaccades and their relationship to multisensory input and integration, attention and oculomotor control, cell recording in animal models is necessary. Monkeys have been used extensively as a model for studying the neurophysiology of the oculomotor and attention systems (Fecteau and Munoz, 2006). While there have been studies on microsaccades in monkeys (Van Gisbergen et al., 1981; Martinez-Conde 2000; Martinez-Conde et al., 2002; Horwitz, et al., 2003; MartinezConde et al., 2006a; Hafed et al., 2009), as well as other animals (Martinez-Conde and Macknik, 2008), to our knowledge there exists only a brief description of microsaccade characteristics during covert orienting using nonpredictive cues (Corneil et al., 2008). Therefore, it is important to verify that equivalent microsaccade behavior is observed in monkeys. Saccade behavior in monkeys during cue-target tasks is consistent with that in humans (Fecteau et al., 2005). We therefore hypothesize that microsaccade metrics will be modulated analogously to what has been observed previously in human experiments. This analogy must be established before commencing detailed neurophysiological studies.

In order to verify the modulation of microsaccade metrics in monkeys, we used cue-target experiments (Figure 1A), similar to that of Posner (1980). In the first set of experiments we investigated the modulation of microsaccades during a purely visual task using predictive and non-predictive visual cues. In a follow up experiment we investigated the modulation of microsaccades during a multisensory cueing task using auditory cues and visual targets. We then compared these results to those found previously in humans. Finally, we present preliminary data showing how the discharge of brainstem omnipause neurons is modulated during the generation of microsaccades. Omnipause neurons are brainstem neurons that act as a gating mechanism for the initiation of saccades (See Scudder et al., 2002 for review). We hypothesize that, if microsaccade modulation is a result of saccade programming during covert attention, omnipause neurons should pause for microsaccades just as they do for larger saccades. 


\section{Methods}

Two male rhesus monkeys (Macaca mulatta) weighing approximately 7 and $10 \mathrm{~kg}$ performed in this study. All protocols were approved by the Queen's University Animal Care Committee and were in full compliance with the Canadian Council on Animal Care policy for the use of laboratory animals.

\section{Cue-target Experiments}

We investigated the effects of visual and auditory cues on microsaccade rates and directions during an oculomotor version of the cue-target task (Figure 1A). Separate analyses from these experiments were previously published to study the neural mechanisms of cueing, independent of microsaccades (Bell et al., 200; Fecteau et al., 2004). Here, we reanalyzed these same data, but now in the context of microsaccade detection and analysis.

Figure 1A depicts the cue-target task used in these experiments. All tasks began with the animal fixating on a fixation point (FP) in the center of a visual screen. After a variable amount of fixation time a cue was flashed in the periphery. After another variable amount of fixation time, the target appeared at the same location as the cue or at a diametrically opposite location. The time between cue and target onset is defined as the cue target onset asynchrony (CTOA). The animal was required to make a saccadic eye movement to the target and was rewarded for doing so. The cue and target appeared left or right and in any combination for all of these tasks with random probability.

Three separate, blocked experiments were performed using variations of the cue-target task. In experiment 1 , the cue and target were visual and the cue did not predict the target (Fecteau et al., 2004). In experiment 2, the cue and target were visual, but the cue predicted the location of the target $75 \%$ of the time (Fecteau et al., 2004). In experiment 3 , the cue was auditory, the target was visual, and the cue was not predictive of the target (Bell et al., 2004). The auditory cue was placed in a position that was commensurate with the position of the visual target. In experiments 1 and 2, the first fixation period was 500$1000 \mathrm{~ms}$ long, the cue was flashed for $30 \mathrm{~ms}$ and CTOAs of $50,100,200,500$ and $1200 \mathrm{~ms}$ were used. In experiment 3 , the first fixation period was $600-800 \mathrm{~ms}$ long, the cue was presented for 50ms and CTOAs of 60, 160 and $610 \mathrm{~ms}$ were used. Note that because all of these experi- ments were originally designed for neurophysiological recording, the actual positions of targets and cues were aligned to the receptive fields of the neurons being studied in the superior colliculus. Therefore, we excluded all blocks of trials where the cue and target locations deviated more than $20^{\circ}$ from the horizontal meridian. This also meant that cue and target eccentricities were variable, ranging from $2-20^{\circ}$ (Figure 1B).
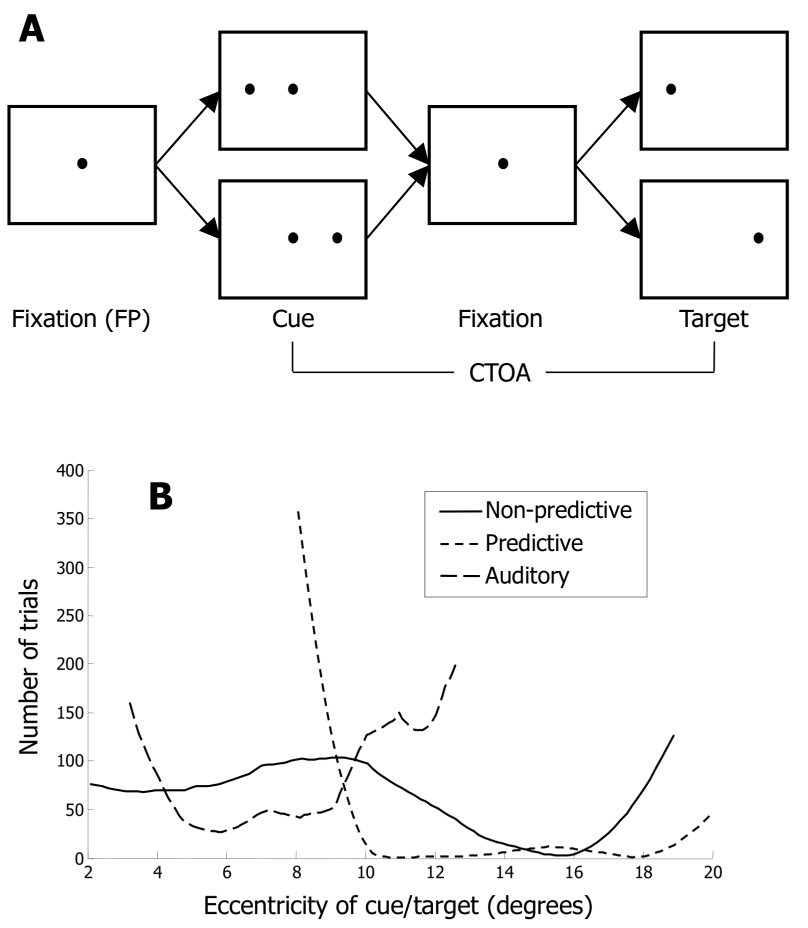

Figure 1. (A) Schematic representation of the cue-target tasks used. Each trial began with the animal fixating on a central fixation point (FP). After a variable time a brief visual or auditory cue was presented on the left or the right. The animal then maintained fixation again for another variable time: the cue-target onset asynchrony (CTOA). The fixation point was then extinguished and a visual target appeared to the left or right at the same eccentricity as the cue. These tasks were preformed in separate experiments such that the animals performed blocks with visual cues that were either nonpredictive or predictive or separate blocks in which auditory cues were non-predictive. (B) Histograms of cue-target eccentricities used in each experiment.

Horizontal and vertical eye position data were recorded with the search coil system and were digitized at $1 \mathrm{KHz}$ for experiments 1 and 2 and $500 \mathrm{~Hz}$ for experiment 3. This technique has a spatial resolution of $0.1^{\circ}$. In our analysis we concentrated on the long CTOA trials $(500 \mathrm{~ms}$ and $1200 \mathrm{~ms}$ for experiments 1 and 2 and $610 \mathrm{~ms}$ for experiment 3) for each experiment. We also only used data 
from trials where the animal performed the task correctly. We detected the microsaccades during the fixation period (i.e., from when the animal first started fixating the central FP until the target appeared) using the algorithm outlined below.

\section{Recording Omnipause Neurons and Microsaccades}

In a separate set of experiments (Everling et al., 1998) we recorded previously from brainstem omnipause neurons (OPNs) in a gap saccade task. Here we describe the discharge of OPNs modulated during the generation of microsaccades from this same data set.

In the gap saccade task, the animal began each trial by fixating on a central FP. In this task there was no cue. After a fixation period of 500-1000 ms the FP disappeared and, following a $200 \mathrm{~ms}$ gap when nothing was present on the screen, a target then appeared $10^{\circ}$ to the left or right. The animal was required to make a saccade to the target. The monkeys performed several blocks of this gap saccade task. Horizontal and vertical eye position data was digitized at $500 \mathrm{~Hz}$. Neuron activity was sampled at $1 \mathrm{kHz}$ after passing through a window discriminator that filtered for action potentials that met both amplitude and temporal constraints. We detected microsaccades during the fixation period using the algorithm outlined below. Data from one monkey is presented.

\section{Microsaccade Detection}

Microsaccades were detected using an algorithm similar to that of Martinez-Conde and colleagues (MartinezConde et al., 2000). We first differentiated the eye position data to produce instantaneous horizontal and vertical velocities. These velocity data were smoothed using a $21 \mathrm{~ms}$ rectangular filter. From the velocity traces we calculated the instantaneous speed and direction at each point. We then thresholded these two values to determine when the eyes were stationary and when they were making a saccade. The instantaneous direction of the eyes could not change more than $15^{\circ}$ to be considered a saccade. The instantaneous speed of the eyes had to be at least $8 \%$ sec. This speed threshold was chosen such that it gave the best main sequence as described by Zuber and colleagues (1965) (see the results section for an example). A saccade was then defined as a sequence where the eye was moving for a period of at least $5 \mathrm{~ms}$. From the eye movement data we then calculated the onset and termination of all saccades, the peak velocity, the saccade vector and the saccade amplitude. Finally, the amplitude was used as a threshold such that we were only interested in microsaccades that were between $0.2-2^{\circ}$.

\section{Microsaccade Analysis}

We analyzed microsaccade direction using a technique developed by Rolfs and colleagues (Rolfs et al., 2005). In order to identify when microsaccade rates differed from randomness significantly over time, we plotted them against randomized data. After removing vertical microsaccades (those greater than $60^{\circ}$ in direction away from horizontal), we shuffled the directions of all microsaccades from the original data, but maintained the microsaccade onsets (i.e., the direction of one microsaccade was randomly assigned to another microsaccade and this was done for each microsaccade). This shuffling of directions maintained any overall biases in direction and any changes in the rate of microsaccades. The process was done 100 times and the rates of congruent and incongruent microsaccades were calculated for each iteration. A congruent microsaccade was one in which the direction was towards the cue location, whereas an incongruent microsaccade was one in which the direction was away from the cue location. A $100 \mathrm{~ms}$ rectangular filter was applied to the individual rates to compensate for splitting the data. The rates were then averaged to get a distribution representing the null hypothesis that there was no significant difference in congruent and incongruent microsaccades. We then calculated the actual rates for congruent and incongruent microsaccades and smoothed those with a $100 \mathrm{~ms}$ rectangular filter. Finally, we plotted the rate deviation of the actually congruent and incongruent microsaccades from the corresponding null distributions (e.g., Figures 3B-6B). We also plotted two standard deviations away from the randomized data (the shaded area of Figures 3B-6B). The rate deviation was significant when it differed from the corresponding randomized data by more than two standard deviations. We only considered deviations that lasted more than $20 \mathrm{~ms}$ as significant.

\section{Results}

We first calculated the main sequence relationship to verify our microsaccade detection algorithm (Figure 2). This figure depicts the expected linear relationship between saccade amplitude and peak velocity of $6112 \mathrm{mi}-$ crosaccades from both monkeys during experiment 1 . 
There was a correlation coefficient of $0.9589(\mathrm{p}<0.5)$ between the two variables and a linear regression to these points had a slope of $0.6868 \mathrm{deg} / \mathrm{sec} /$ minarc with an intercept of $4.4182 \mathrm{deg} / \mathrm{sec}$. These results verify the algorithm, based on the fact that microsaccades follow the same amplitude-velocity relationship as larger saccades (Zuber et al., 1965).

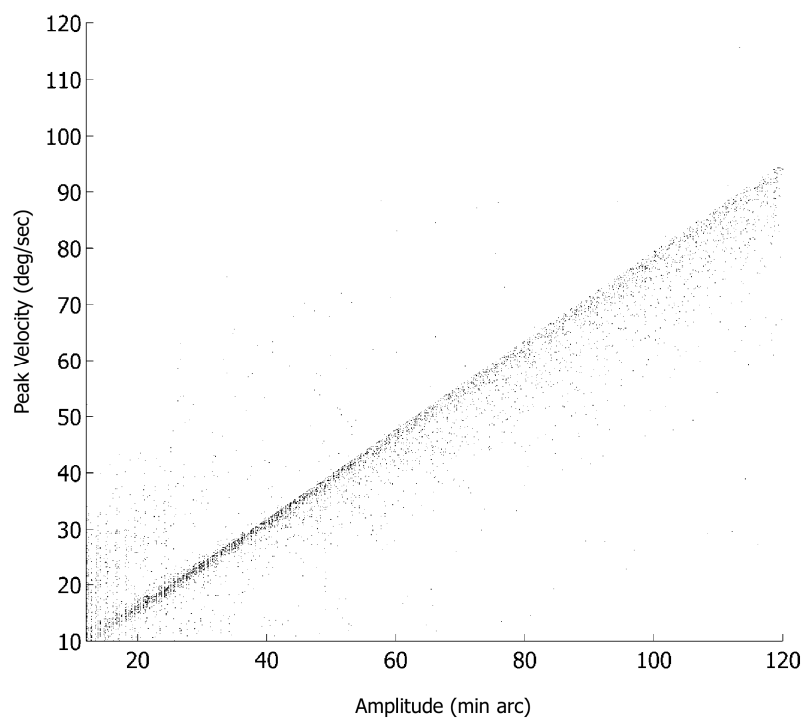

Figure 2. The main sequence plot for monkey A collapsed over all visual cueing experiments. The expected linear relationship between amplitude and peak velocity was observed.

\section{Experiment 1: non-predictive visual cues}

Behavior. In this experiment, cue location did not predict target location. See Fecteau et al., (2004) for the original analysis of behavior. At the short CTOAs (50ms), saccadic reaction times were significantly shorter when the cue appeared at the same location as the target, compared to when the cue and target appeared on opposite sides. This has been called attention capture. However, at longer CTOAs (100-1200ms), saccadic reaction times were significantly longer when the cue appeared at the same location as the target. This has been referred to as inhibition or return.

Microsaccade statistics. Figure 3A summarizes the average microsaccade rate from 2 monkeys during the fixation period of the visual non-predictive cueing task. The monkeys performed 5190 trials and 4275 microsaccades were detected. The rate for each animal was also smoothed using a $10 \mathrm{~ms}$ rectangular filter. There was an over-representation of microsaccades early in the fixation epoch before cue appearance that was likely due to small corrective saccades after the animal reached the fixation window around the central FP. Just before the cue appeared there was a stable baseline rate of about 0.5 saccades/s. Following cue onset, microsaccade rate began to decrease after about $50 \mathrm{~ms}$ and reached a minimum of about 0.2 saccades/s during the interval $120-250 \mathrm{~ms}$ after cue onset. The rate then began to increase again and reached a peak of about 0.7 saccades/s $400 \mathrm{~ms}$ after cue onset. We chose 3 epochs to test these rates quantitatively. t1 refers to a $50 \mathrm{~ms}$ epoch before the onset of the cue. $\mathrm{t} 2$ refers to aa epoch $100-150 \mathrm{~ms}$ after onset of the cue and is used to test for a decrease in microsaccade rate soon after cue onset. $\mathrm{t} 3$ refers to an epoch $400-450 \mathrm{~ms}$ after onset of the cue and is used to test for an increase in microsaccade rate after cue onset. Using a t-test we found a significant decrease in microsaccade rate during $\mathrm{t} 2$, when compared to $\mathrm{t} 1$ ( $\mathrm{p}<0.5$ ), and a significant increase in microsaccade rate during $\mathrm{t} 3$, when compared to $\mathrm{t} 1$ ( $\mathrm{p}<$ $0.5)$.

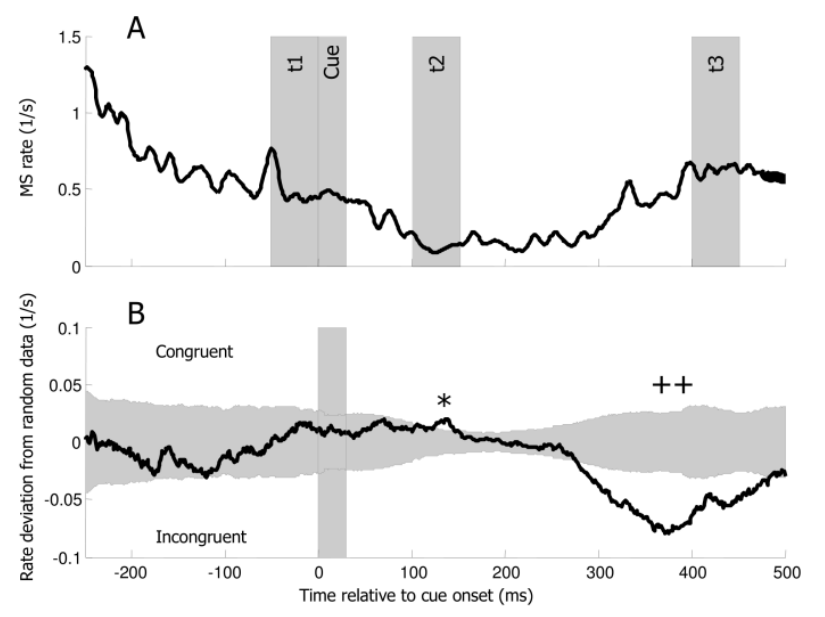

Figure 3. The modulation of microsaccade rates during fixation in a visual non-predictive cue-target task. Average microsaccade rate is plotted relative to cue onset ( $A$ ). $t 1, t 2$ and t3, refer to time epochs chosen to analyze changes in rate after stimulus presentation. The rate deviation of congruent and incongruent microsaccades from randomized data is also plotted $(B)$. Congruency refers to cue and microsaccade direction. The shaded area represents two standard deviations from the mean of 100 randomized data sets. Hence, when the rate deviation is greater than or larger than the shaded area, the deviation is significant.

Two significant deviations from the randomized data were observed for non-predictive visual cues. The first significant difference occurred 112-150 ms after cue appearance (see * in Figure $3 \mathrm{~B}$ ). During this period there 
was a significant increase in cue-congruent microsaccades and a brief, but significant decrease in cueincongruent microsaccades (i.e., microsaccades tended to point in the direction of the cue). The second significant differences occured $280-475 \mathrm{~ms}$ after the cue (see ++ in Figure 3B). During this time there was a significant decrease in cue-congruent microsaccades and a significant increase in cue-incongruent microsaccades (i.e., microsaccades tended to point in the opposite direction of the cue).

\section{Experiment 2: predictive visual cues}

Behavior. In this experiment, cue location was predictive of target location on $75 \%$ of trials. See Fecteau et al., 2004 for the original analysis of behavior. For targets appearing at the same location as the cue, it was found that predictive cues enhanced the effects of capture of attention. Specifically, saccadic reaction times were faster for predictive cues than for non-predictive cues and this enhancement effect was present for all but the longest CTOA (1200 ms). For targets appearing at the opposite location to the cue, this enhancement effect was not present. However, for the $50 \mathrm{~ms}$ CTOA, saccadic reaction times were faster for non-predictive cues than for predictive cues.

Microsaccade statistics. Figure 4A summarizes the average microsaccade rate for blocks of trials utilizing predictive cues. 6302 trials were performed between the two animals and 3306 microsaccades were detected. Similar to the non-predictive cues, we observed a baseline of about 0.5 saccades/s before cue presentation. Following cue presentation, microsaccade rate started to decline at about $80 \mathrm{~ms}$ and reached a minimum of about 0.05 saccades/s at $120-200 \mathrm{~ms}$. The rate then began to increase steadily until it reached a peak of about 0.7 saccades/s at approximately $450 \mathrm{~ms}$. Again, we found a significant decrease in microsaccade rate during $\mathrm{t} 2$, when compared to $\mathrm{t} 1 \quad(\mathrm{p}<0.5)$, and a significant increase in microsaccade rate during $\mathrm{t} 3$, when compared to $\mathrm{t} 1 \mathrm{p}<$ $0.5)$.

To study microsaccade directions we performed the null distribution analysis. Similar deviations from randomness to the non-predictive cueing task could be observed during the predictive cueing task (Figure 4B). Specifically, there was an early significant increase in congruent saccades and consequently a significant decrease in incongruent saccades at $50-150 \mathrm{~ms}$ (see $*$ in
Figure 4B). This was followed by a later and more pronounced significant decrease in congruent saccades and significant increase in incongruent saccades at approximately 280-500 ms (see ++ in Figure 4B).
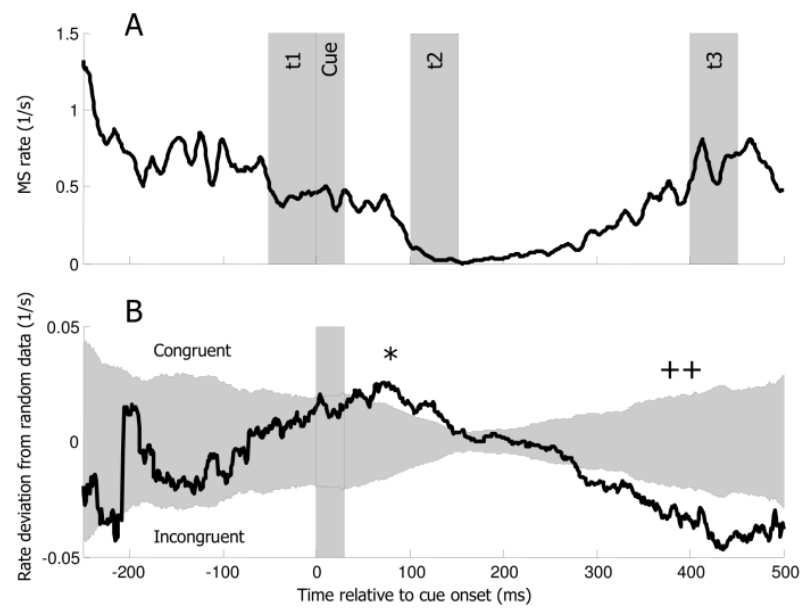

Figure 4. The modulation of microsaccade rates during fixation in a visual predictive cue-target task. This figure was created using the same analysis as in Fig. 3.

\section{Experiment 3: non-predictive auditory cues}

Behaviour. We also employed auditory cues to determine their effect on microsaccade production. See Bell et al., 2004 for the original analysis of behavior. In contrast to what was observed for predictive and nonpredictive visual cues, the auditory cues we used preceding a visual target did not have any significant effect on reaction time for either short or long CTOAs.

Microsaccade statistics. Microsaccade rates for auditory cues (Figure 5A) were analyzed in the same manner as for visual cues. 778 trials were performed between the two animals and 704 microsaccades were detected. The baseline microsaccade rate was about 0.8 saccades/s before cue presentation. The rate began to decline 20-30 ms after cue presentation and reached a minimum of 0.3 about saccades/s at 150-200 ms after cue onset. The rate then increased and reached a maximum of about 1.1 saccades/s at 450ms. Again, we found a significant decrease in microsaccade rate during $\mathrm{t} 2$, when compared to $\mathrm{t} 1$ ( $\mathrm{p}<$ 0.5 ), and a significant increase in microsaccade rate during $\mathrm{t} 3$, when compared to $\mathrm{t} 1(\mathrm{p}<0.5)$.

Microsaccade directions were again analyzed using the null distribution method. No significant deviations from randomness could be observed for either congruent or incongruent microsaccades (Figure 5B), but there were 
late trends of decreased congruent microsaccades and increased incongruent microsaccades at 250-450 ms after cue onset, which was consistent with the trends observed in the visual cueing experiments. To further investigate this trend, we split the congruent and incongruent data sets into their respective left and right cue conditions (Figure 6). In this analysis, we observed that left auditory cues were responsible for this late trend towards incongruent microsaccades. This trend was significant for left cues at approximately 350-425 ms, but did not exist for right cues (see ++ in Figure 6).

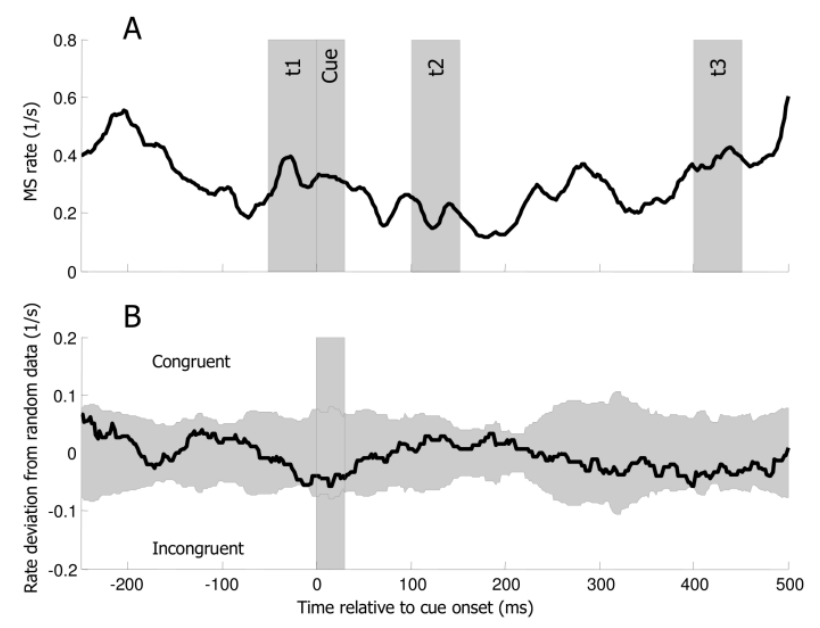

Figure 5. The modulation of microsaccade rates during fixation in an auditory non-predictive cue-target task. This figure was created using the same analysis as in Fig. 3.

\section{Experiment 4: recording from omnipause neurons}

We had the opportunity to assess the activity of brainstem OPNs during microsaccade generation by reanalyzing data that was published previously (Everling et al., 1998). OPNs are tonically active for fixation and pause for all macrosaccades, thereby gating the occurrence of saccades (see Scudder et al., 2002 for review). Our goal here was to determine whether OPNs also paused for microsaccades. We recorded from 18 OPNs in one monkey and collapsed the data. The animal performed 1306 trials and we identified 250 microsaccades. The lower number of microsaccades was likely due to the limited fixation period analyzed and the absence of a cue. Figure 7 summarizes the activity of OPNs aligned on microsaccade onset for those microsaccades where there was at least $100 \mathrm{~ms}$ of fixation before and after microsaccade occurrence (177 trials). We identified two time periods to analyze the firing rate of OPNs. The first epoch, $\mathrm{t} 1$, refers to $35-50 \mathrm{~ms}$ before microsaccade onset and was used as the baseline firing rate. The second epoch, $\mathrm{t} 2$, refers to $0-15 \mathrm{~ms}$ after microsaccade onset. The baseline firing rate was 162 spikes/s (t1) whereas the rate directly after microsaccade onset was 62 spikes/s (t2). A t-test revealed that this difference was significant ( $\mathrm{p}<0.05)$. Thus, OPNs pause for microsaccades just as for larger macro saccades (i.e. $>2^{\circ}$ amplitude).

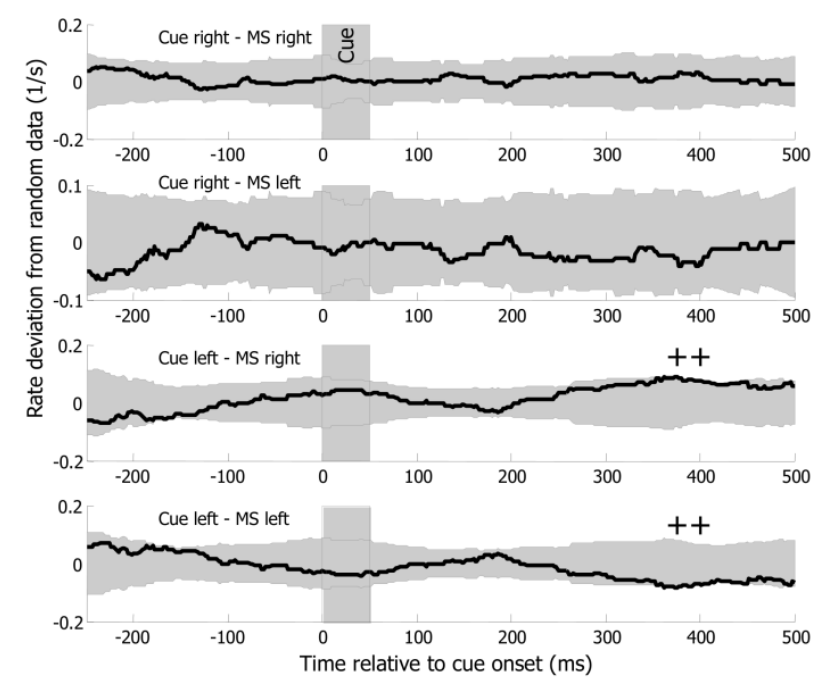

Figure 6. The modulation of horizontal microsaccade rates during fixation in an auditory non-predictive cue-target task. The rate deviation of left and right microsaccades from randomized data during left and right cues is plotted. This figure was created using the same analysis as in Fig. 3.

\section{Discussion}

Using peripheral visual and auditory cues in a cuetarget paradigm, we have shown that microsaccade properties are modulated during reflexive and voluntary covert shifts of visual attention in monkeys. Microsaccade rates decreased quickly after cue presentation, reaching a minimum approximately $100 \mathrm{~ms}$ later. This early decrease of microsaccade frequency was followed by a later enhancement, reaching a maximum approximately 400 ms after cue presentation. This early drop in rate is similar to saccade inhibition produced by transient changes in the visual display (Reingold and Stampe, 2002). Microsaccade directions were also biased by cue presentation. For visual cues there was an early cue congruent bias in microsaccades, followed by a later and more pronounced 
cue incongruent bias. For auditory cues there was only a cue incongruent bias and only for cues to the left.

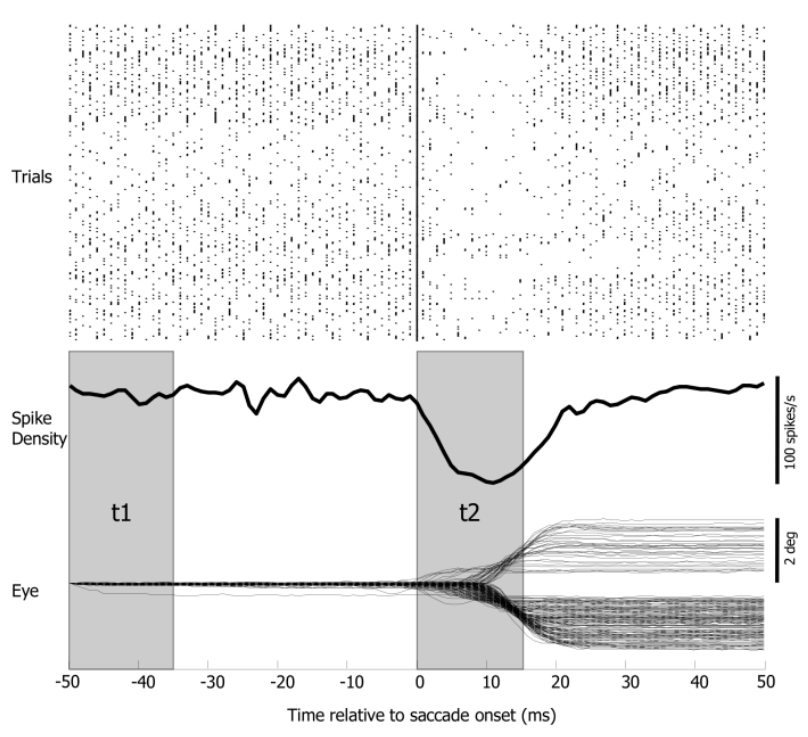

Figure 7. The modulation of omnipause neuron activity during microsaccade generation. The individual action potentials for 177 trials are plotted at the top. Followed by the spike density function representing the relative average number of spikes (convolved with a Gaussian function of width $10 \mathrm{~ms}$ ) and the eye position traces of microsaccades. 11 refers to a period 35-50ms before microsaccade onset and was used to calculate a baseline rate of neuronal activity. $t 2$ refers to a period $0-15 \mathrm{~ms}$ after microsaccade onset and was used to calculate the rate of neuronal activity during microsaccades.

Several studies have investigated the effects of covert orienting on microsaccades in humans using cue-target paradigms. Our results from monkeys are largely in agreement with the results of previous human studies. The modulation of microsaccades rates following cue presentation was first observed in humans by Engbert and Kliegl (2003) using predictive, endogenous visual cues. Immediately following cue presentation, they observed an inhibition of microsaccades to $20 \%$ of baseline, reaching a minimum approximately $150 \mathrm{~ms}$ later. Following this, they observed an enhancement of microsaccades to double the baseline rate, reaching a maximum approximately $350 \mathrm{~ms}$ after cue presentation. This characteristic pattern was also seen in monkeys (Figures 3, 4), although the inhibition appeared to occur earlier and reach a minimum earlier than in the human studies. They also found a baseline rate of 1 saccade/s during fixation, whereas we found a baseline rate closer to 2 saccades/s. The differences in time course and baseline rates could be due to many factors, such as experimental condition, species differences or extensive overtraining in the monkeys, but the overall pattern was the same. They also found that this modulation occurred during neutral cues that did not convey any information about the target. They concluded that any visual transient was sufficient to produce this result. This was supported by subsequently studies that found this characteristic modulation regardless of the type or meaning of the cue (Galfano, 2004 - nonpredictive exogenous visual cues; Laubrock, 2004 - nonpredictive endogenous and exogenous visual cues; Rolfs et al., 2004 - predictive exogenous, visual and auditory cues; Rolfs et al., 2007 - predictive visual cues). Our results are consistent with these findings because we observed this modulation in both predictive and non predictive cues, as well as with auditory cues.

Several studies have examined the effects of visual cues on microsaccade direction. Hafed and Clark (2002) first observed the characteristic pattern of a cuecongruent microsaccade bias soon after the presentation of a predictive cue, followed by a cue-incongruent microsaccade bias. They correlated these biases in microsaccade direction with behavioral differences and suggested that microsaccades were influenced by covert attentional shifts. Their experimental set up used a cue that was always on and involved multiple targets on the horizontal and vertical meridian. Despite the differences between their task and our horizontal cue-target tasks, our results are consistent with theirs. In separate studies, Laubrock and colleages (2005) and Rolfs and colleages (2005) used cue-target paradigms with predictive, transient visual cues and also found this characteristic pattern. Both studies identified an early cue-congruent bias at approximately 50-150 ms after cue presentation, followed later by a cue-incongruent bias at approximately 250-550 ms. We found similar epochs in our results for predictive visual cues (Figure 4). Galfano and colleagues (2004) used peripheral, non-predictive visual cues, but while they did find the cue-incongruent bias at $300 \mathrm{~ms}$ after cue presentation, they did not observe the early cue-congruent effect that we found in our non-predictive visual cueing task (Figure 3). This could be the result of species differences or the amount of training that monkeys underwent prior to data collection. This may also be the result of different task conditions. First, we use flashed points for our cues, while Galfano and colleagues (2004) used boxes that were always on and became thicker and more intense when cued. Hence, it is possible that our cues elicited a stronger capture of attention response. Second, 
we use saccades to targets to signify a response and measure behavior, while they used manual button press responses. Because the monkeys were required to move their eyes towards the target, the effect of the visual cue may have been enhanced.

Support for visual cues enhancing covert attention responses in microsaccades comes from Rolfs and colleagues (2005) who also observed the effects of auditory cues on microsaccade direction. They found that in a purely auditory task (i.e., auditory cues and targets) there were no early cue-congruent or late cue-incongruent biases. There was only a cue-congruent bias that happened later, and only for left cues, around $200 \mathrm{~ms}$ after target presentation. They argued that this was a result of decreased occulomotor control resulting from a lack of visual information in the purely auditory tasks. Accordingly, when human subjects performed a task with auditory cues and visual targets, they observed a cueincongruent bias $300-400 \mathrm{~ms}$ after cue presentation for cues to the left. These results agree with our findings for auditory cues and visual targets in monkeys, where we found cue-incongruent biases for cues to the left at a similar epoch (Figure 6). Rolfs and colleagues (2005) suggested that this left-hemisphere bias could be explained by a left-hemisphere advantage to brief auditory cues (Brown and Nicholls, 1997, Nicholls et al., 1999) or by a right-hemisphere bias of attention (Reuter-Lorenz et al., 1990).

We provide further support that microsaccades reflect the orientation of covert spatial attention. As in studies with humans, we observed microsaccade directions that were consistent with an early capture of attention and a later inhibition of return. Fecteau and colleagues (2004) observed the largest capture of attention effect in monkeys at the $50 \mathrm{~ms}$ CTOA in both predictive and nonpredictive visual cueing. During this time period we saw a significant bias in cue-congruent microsaccades. For CTOAs of 100-1200 ms they saw an inhibition of return response in both experiments that peaked at a CTOA of $200 \mathrm{~ms}$ and then gradually declined. During this time period we saw a significant bias in cue-incongruent microsaccades. Both microsaccade rate and direction modulations in monkeys during cue-target tasks are consistent with that found in human studies. From these results we conclude that monkeys are a suitable model for studying the neurological pathways involved in the generation and manipulation of microsaccades. Future experiments can use cell recording to elucidate these pathways and how the activation of the visual system during covert orienting influences the behaviour of microsaccades.

We examined the modulation of brainstem OPNactivity during microsaccade generation (Figure 7). Hafed and Clark (2002) suggested that because microsaccades follow the same velocity-amplitude relationship as larger saccades (Zuber et al., 1965) and reflect the orientation of covert visual attention, it is likely that the same neurocircuitry governing larger saccades is responsible for the generation and modulation of microsaccades. One component of this circuitry is OPNs, which are brainstem neurons that are tonically active and only pause during saccade generation (Cohen and Henn 1972, Keller 1974, Luschei and Fuchs 1972). Specifically, OPNs are tonic inhibitors of burst neurons located in the paramedian pontine reticular formation and the rostral interstitial nucleus of the medial longitudinal fasciculus (see Fuchs et al., 1985 for a review). These burst neurons produce high-frequency discharges during saccades and thus have the opposite discharge pattern of OPNs. Hence, OPNs act to stabilize the visual system and act as a gating mechanism for the generation of saccades (See Scudder et al., 2002 for a review). It is reasonable then that they would also gate microsaccades and indeed, this is what we observed. OPNs paused for microsaccades (Figure 7) as they do for larger saccades. Everling and colleagues (1998) observed that OPNs displayed a small burst in activity approximately $60 \mathrm{~ms}$ after target presentation during regular-latency saccades. This burst occurs approximately the same time that we observed the overall microsaccade rate to decrease after cue presentation. It is possible that increased OPN activity during this time triggered the decrease in microsaccade rate. Finally, OPNs were also implicated by Ashe et al., (1991) as a cause for microsaccadic flutter, which is a rare disorder characterized by saccadic oscillations. From these findings it seems likely that OPNs are involved in governing microsaccades.

Van Gisbergen and colleagues (1981) found that burst neurons and motoneurons burst or paused in time with microsaccades, supporting the hypothesis that burst neurons are controlling the generation and inhibition of microsaccades. More recently, Hafed and colleagues (2009) found that, during a fixation task, neurons in the rostral pole of the superior colliculus (SC) burst before and during microsaccades and were selective for the amplitude 
and direction of these saccades. They also found that inactivation of these neurons resulted in a decrease in microsaccade rate.

Behavioural studies have also provided insight into possible neural mechanisms governing microsaccades. Rolfs and colleagues (Rolfs et al., 2006) showed, in visual and memory guided tasks, that saccadic reaction time was increased when microsaccades occured within 150 $\mathrm{ms}$ of a required saccade. They further showed that microsaccade rate decreases steadily towards zero before saccade onset. They hypothesized that this provides support for microsaccades resulting from activity in the rostral pole of the SC, since larger saccades have to overcome this fixation activity before they are executed. Based on this idea, Rolfs and colleagues (Rolfs et al., 2008 b) went on to postulate a common-field model for microsaccade and saccade generation. This model also predicted that saccadic reaction time would be correlated with the amplitude of microsaccades occurring directly before the saccade and they indeed did find this in their data (Rolfs et al., 2008a). However, Valsecchi and Turatto (Valsecchi and Turatto, 2007) showed that microsaccade rate responded similarly when stimuli were visible and invisible to the SC, suggesting that other brain areas are involved in microsaccade generation. Finally, OteroMillan and colleagues (Otero-Millan et. al 2008) showed that saccades and microsaccades have equivalent spatiotemporal characteristics during visual exploration, search and prolonged fixation. The results of these behavioural and neurophysiological studies, together with our results on omnipause neurons, suggest a common mechanism for the generation of microsaccade and larger saccades. Thus, biases in these neural mechanisms, such as those generated by shifts in visual attention, should modulate both microsaccades and larger saccades.

In summary, we have verified that microsaccade metrics in monkeys are modulated by reflexive covert attention analogously to what has been found in human studies. Consequently, we can use monkeys as a model for studying the neurophysiology of microsaccades. We have provided further evidence that microsaccade orientation reflects the orientation of covert visual attention. Finally, we have provided evidence that the same neurocircuitry is involved in the generation of microsaccades and larger saccades. Future experiments in monkeys will involve cell recording and will require longer fixation periods to generate an accurate picture of the time course of microsaccade production.

\section{Acknowledgements}

We thank Ann Lablans for outstanding technical assistance and members of the Munoz lab for commenting on an earlier draft of the manuscript. This work was supported by research grants from the Canadian Institutes of Health Research. Douglas P. Munoz was supported by the Canada Research Chair Program.

\section{References}

Ashe, J., Hain, T. C., Zee, D. S., \& Schatz, N. J. (1991). Microsaccadic flutter. Brain, 114, 461-472.

Barlow, H. B. (1952). Eye movements during fixation. Journal of Physiology, 116, 290-306.

Bell, A. H., Fecteau, J. H., \& Munoz, D. P. (2004). Using auditory and visual stimuli to investigate the behavioral and neuronal consequences of reflexive covert orienting. Journal of Neurophysiology, 91, 21722184.

Brown, S. \& Nicholls, M.E. (1997). Hemispheric asymmetries for the temporal resolution of brief auditory stimuli. Perception \& Psychophysics, 59, 442-447.

Cohen, B. \& Henn, V. (1972). Unit activity in the pontine reticular formation associated with eye movements. Brain Research, 46, 403-410.

Coppola, D. \& Purves, D. (1996). The extraordinarily rapid disappearance of entopic images. Proceedings of the National Academy of Sciences, 93(15), 80018004.

Corneil, B. D., Munoz, D. P., Chapman, B. B., Admans, T. \& Cushing, S. L. (2008). Neuromuscular consequences of reflexive covert orienting. Nature Neuroscience, 11(1), 13-15.

Ditchburn, R. W., Fender, D. H., \& Mayne, S. (1959). Vision with controlled movements of the retinal image. Journal of Physiology, 145, 98-107.

Engbert, R. \& Kliegl. (2003). Microaccades uncover the orientation of covert attention. Vision Research, 43, 1035-1045. 
Everling, S., Pare, M., Dorris, M. C., \& Munoz, D. P. (1998). Comparison of the discharge characteristics of brain stem omnipause neurons and superior colliculus fixation neurons in monkey: implications for control of fixation and saccade behavior. Journal of Neurophysiology, 79, 511-528.

Fecteau, J. H., Bell, A. H., \& Munoz, D. P. (2004). Neural correlates of the automatic and goal-driven biases in orienting spatial attention. Journal of Neurophysiology, 92, 1728-1737.

Fecteau, J. H. \& Munoz, D. P. (2005). Correlates of capture of attention and inhibition of return across states of visual processing. Journal of Cognitive Neuroscience, 17(11), 1714-1727.

Fecteau, J. H. \& Munoz, D. P. (2006). Salience, relevance, and spiking neurons: a priority map governs target selection. Trends in Cognitive Science, 10, 382390.

Fuchs, A. F., Kaneko, C. R. S. \& Scudder, C. A. (1985). Brainstem control of saccadic eye movements. Annual Review of Neuroscience, 307-337.

Galfano, G., Betta, E., \& Turatto, M. (2004). Inhibition of return in microsaccades. Experimental Brain Research, 159, 400-404.

Hafed, Z.M. \& Clark, J.J. (2002). Microsaccades as an overt measure of covert attention shifts. Vision Research, 42, 2533-2545.

Hafed, Z.M., Goffart, L. \& Krauzlis, R.J. (2009). A Neural Mechanism for Microsaccade Generation in the Primate Superior Colliculus. Science, 323, 940943.

Horwitz, G. D., \& Albright, T. D. (2003). Short-latency fixational saccades induced by luminance increments. Journal of Neurophysiology, 90, 1333-1339.

Horowitz, T. S., Fine, E. M., Fencsik, D. E., Yurgenson, S. \& Wolfe, J. M. (2007a). Fixational eye movements are not an index of covert attention, Psycological Science, 18, 356-363.

Horowitz, T. S., Fine, E. M., Fencsik, D. E., Yurgenson, S. \& Wolfe, J. M. (2007b). Microsaccades and attention. Does a weak correlation make an index? Reply to Laubrock, Engbert, Rolfs, and Kliegl. Psycological Science, 18, 367-368.
Jonides, J. (1981). Voluntary vs. automatic control over the mind's eye's movement. In J. Long \& A. Baddeley (Eds.), Attention and Performance IX (pp. 187203). Hillsdale: Lawrence Erlbaum Associates.

Keller, E. (1974). Participation of the medial pontine reticular formation in eye movement generation in the monkey. Journal of Neurophysiology, 37, 316-332.

Kliegl, R., Rolfs, M., Laubrock, J. \& Engbert, R. (2009). Microsaccadic modulation of response times in spatial attention tasks. Psychological Research, 73, 136-146.

Laubrock, J., Engbert, R., \& Kliegl, R. (2004). Microsaccade dynamics during covert attention. Vision Research, 45, 721-730.

Laubrock, R., Engbert, R., Rolfs, M. \& Kliegl, R. (2007). Microsaccades are an index of covert attention. Psycological Science, 18, 364-366.

Lushei, E.S. \& Fuchs, A.F. (1972). Activity of brain stem neurons during eye movements of alert monkeys. Journal of Neurophysiology, 35, 445-461.

Martinez-Conde, S., Macknik, S. L., \& Hubel, D. H. (2000). Microsaccadic eye movements and firing of single cells in the striate cortex of macaque monkeys. Nature Neuroscience, 3(3), 251-258.

Martinez-Conde, S., Macknick, S. L., \& Hubel, D. H. (2002). The function of bursts of spikes during visual fixation in the awake primate lateral geniculate nucleus and primary visual cortex. Proceedings of the National Academy of Sciences, 99(21), 13920-13925.

Martinez-Conde, S., Macknik, S. L., \& Hubel, D. H. (2004). The role of fixational eye movements in visual perception. Nature Reviews Neuroscience, 5, 229240.

Martinez-Conde, S. (2006a). Fixational eye movements in normal and pathological vision. Progress in Brain Research, 154, 151-176.

Martinez-Conde, S., Macknik, S. L., Troncoso, X. G., \& Dyar, T. A. (2006b). Microsaccades counteract visual fading during fixation. Neuron, 49, 297-305.

Martinez-Conde, S. \& Macknik, S. L. (2007). Windows on the Mind. Scientific American, 297(2), 56-63.

Martinez-Conde, S. \& Macknik, S. L. (2008). Fixational eye movements across vertebrates: Comparative dynamics, physiology, and perception. Journal of $\mathrm{Vi}$ sion, 8(14), 1-16. 
Martinez-Conde, S., Macknik, S. L., Troncoso, X. G. \& Hubel, D. H. (2009). Microsaccades: a neurophysiological analysis. Trends in Neurosciences, 32(9), 463475 .

Maylor, E. A., \& Hockey R. (1985). Inhibitory component of externally controlled covert orienting in visual space. Journal of Experimental Psychology: Human Perception and Performance, 11, 777-787.

Nicholls, M. E., Schier, M., Stough, C.K., \& Box, A. (1999). Psychophysical and electrophysiological support for a left hemisphere temporal processing advantage. Neuropsychiatry, Neuropsychology, \& Behavioral Neurology, 12, 11-16.

Otero-Millan, J, Troncoso, X. G., Macknik, S. L., Serrano-Pedraza, I. \& Martinez-Conde, S. (2008). Saccades and microsaccades during visual fixation, exploration, and search: Foundations for a common saccadic generator. Journal of Vision, 8(14):21, 1-18.

Posner, M. I. (1980). Orientation of attention. The VIIth Sir Frederic Bartlett lecture. Quarterly Journal of Experimental Psychology, 32A, 3-25.

Posner, M. I., \& Cohen, Y. (1984). Components of visual orienting. In H. Bouma \& D. G. Bouwhuis (Eds.), Attention and performance $X$ (pp. 531-556). Hillsdale: Lawrence Erlbaum Associates.

Posner, M. I., Rafal, R. D., Choate, L. S., \& Vaughan, J. (1985). Inhibition of return: neural basis and function. Cognitive Neuropsychology, 2, 221-228.

Ratliff, F., \& Riggs, L. A. (1950). Involuntary motions of the eye during monocular fixation. Journal of Experimental Psychology, 40, 687-701.

Reingold, E. M., \& Stampe, D. M. (2002). Saccadic inhibition in voluntary and reflexive saccades. Journal of Cognitive Neuroscience, 14, 371-388.

Remington, R. W., Johnston, J. C. \& Yantis, S. (1992). Involuntary attentional capture by abrupt onsets. Percept Psychophys, 51, 279-290.

Reuter-Lorenz, P.A., Jha, A.P. \& Rosenquist, J.N. (1990). Hemispheric control of spatial attention. Brain and Cognition, 12, 240-266.

Riggs, L. A., Ratliff, F., Cornsweet, J. C., \& Cornsweet, T. N. (1953). The disappearance of steadily fixated test objects. Journal of the Optical Society of America, 43, 495-501.
Rolfs, M., Engbert, R., \& Kliegl, R. (2004). Microsaccade orientation supports attentional enhancement opposite a peripheral cue. Commentary on Tse, Sheinberg, and Logothetis (2003). Psychological Science, $15,705-707$.

Rolfs, M., Engbert, R., \& Kliegl, R. (2005). Crossmodal coupling of oculomotor control and spatial attention in vision and audition. Experimental Brain Research, $166,427-439$.

Rolfs, M., Laubrock, J. \& Kliegl, R. (2006). Shortening and prolongation of saccade latencies following microsaccades. Experimental Brain Research, 169, 369376.

Rolfs, M., Laubrock, J \& Kliegl, R. (2008a). Microsaccade-induced prolongation of saccade latencies depends on microsaccade amplitude, Journal of Eye Movement Research, 1(3), Art. 5, 1-8.

Rolfs, M., Kliegl, R. \& Engbert, R. (2008b). Toward a model of microsaccade generation: The case of microsaccadic inhibition, Journal of Vision 8, (11), Art. $5,1-23$.

Rolfs, M. (2009). Microsaccades: Small steps on a long way. Vision Research, 49, 2415-2441.

Scudder, C. A., Kaneko, C. S., \& Fuchs, A. F. (2002). The brainstem burst generator for saccadic eye movements. A modern synthesis. Experimental Brain Research, 142, 439-462.

Steinman, R. M., Haddad, G. M., Skavenski, A. A., \& Wyman, D. (1973). Miniature eye movements. Science, 181, 810-819.

Troncoso, X. G., Macknik, S. L. \& Martinez-Conde, S. (2008). Microsaccades counteract perceptual fillingin. Journal of Vision, 8(14), 1-9.

Van Gisbergen, J. A. M., Robinson, D. A. \& Gielen, S. (1981) A Quantitative Analysis of Generation of Saccadic Eye Movements by Burst Neurons. Journal of Neurophysiology, 45(3), 417-438.

Valsecchi, M. \& Turatto, M. (2007). Microsaccadic response to visual events that are invisible to the superior colliculus. Behavioral Neuroscience, 121, 786793.

Zuber, B. L., Stark, L., \& Cook, G. (1965). Microsaccades and the velocity-amplitude relationship for saccadic eye movements. Science, 150, 1459-1460. 